\title{
LPG CONCENTRATION MEASUREMENT IN VEHICLES TRANSPORTING DANGEROUS GOODS
}

This paper describes the design of intelligent sensor unit for LPG (Liquefied Petroleum Gas) concentration measurement in the air. The sensor unit is a part of sensor network which has been designed for vehicles transporting dangerous goods. The described sensor unit uses sensor element based on $\mathrm{SnO}_{2}$ structure which is vapoured on the aluminum substrate. Main part of the paper is focused on hardware solution of the sensor unit, signal processing applied on signal from its sensor element, compensation of air temperature and relative humidity influence on the sensor resistance and description of its application layer communication protocol.

\section{Basic Conception of Inteligent Sensor Unit}

The presented sensor unit is designed as an autonomous subsystem which is able to inform the onboard unit (OBU) - about concentration of LPG in ambient air. The sensor unit consists of analog sensor elements whose output signals are processed by microcomputer in real time (Fig. 1). The result values are transferred into master unit by using of CAN bus [1] and proprietary communication protocol on the application layer.

For LPG concentration measurement the sensor Figaro TGS 813 [6] based on the $\mathrm{SnO}_{2}$ structure vapoured on aluminum substrate was selected. The LPG influences the resistance of sensor. This influence can be modeled by formula (1), where $A, \alpha$ are constants for given type of LPG sensor, $C$ is LPG concentration (in ppm) and $R_{S 20}$ is sensor resistance at temperature $20{ }^{\circ} \mathrm{C}$ and relative humidity $65 \%$. Moreover, the value of sensor resistance depends on air temperature and relative humidity. For compensation of their influences the sensor unit is equipped with temperature sensor and relative humidity sensor. The compensation of LPG concentration is being performed in real time by microcomputer software which uses mathematical apparatus described in next text.

$$
R_{S 20}=A \cdot C^{-\alpha}
$$

\section{Measurement of LPG Concetration}

The LPG sensor element is connected into the microcomputer in accordance with schematics in Fig. 2. The LPG in ambient air influences its resistance so that measurement of LPG concentration is changed to measurement of sensor resistance. Therefore the sensor element is connected as a part of voltage divider with variable ratio of division together with resistor $R_{M}$. Input of the divider is connected to the supply voltage $U_{C C}$. The voltage $U_{R M}$ from resistor $R_{M}$ is connected to the input of $\mathrm{A} / \mathrm{D}$ converter whose reference voltage is connected to $U_{C C}$, too. Therefore the resistance of sensor element can be calculated from formula (2). Voltage $U_{R M}$ is measured by the $\mathrm{A} / \mathrm{D}$ converter with resolution $r b$ according to formula (3) where $R E S_{A D 0}$ is the result of $\mathrm{A} / \mathrm{D}$ conversion. Combining the formulae (2) and (3) the sensor resistance $R_{S}$ can be calculated from formula (4). Note that the formula (4) is independent on supply voltage $U_{C C}$. This fact has positive influence on precision of measurement.

$$
\begin{aligned}
& R_{S}=R_{M} \cdot \frac{U_{C C}-U_{R M}}{U_{R M}} \\
& U_{R M}=R E S_{A D 0} \cdot \frac{U_{C C}}{2^{r b}-1} \\
& R_{S}=R_{M} \cdot \frac{\left(2^{r b}-1\right)-R E S_{A D 0}}{R E S_{A D 0}}
\end{aligned}
$$

The value of LPG concentration (in ppm) can be calculated according to formula (5) which comes out from (1). In the formula (5) the $R_{S 20}$ is resistance of sensor element after compensation of temperature and relative humidity influence. This compensation is given by formula (14). The constant $R_{0}$ is resistance of sensor element at $C=1800 \mathrm{ppm}, T_{M E A S}=20{ }^{\circ} \mathrm{C}$ and $R H=65 \% . K$ and $\alpha$ are specific constants for the given type of sensor.

$$
C=10 \frac{\log \left(\frac{R_{S 20}}{R_{e} \cdot K}\right)}{\alpha}
$$

\footnotetext{
* Martin Vestenický ${ }^{1}$, Peter Vestenický ${ }^{2}$

${ }^{1}$ Department of Telecommunications and Multimedia, Faculty of Electrical Engineering, University of Zilina, Slovakia,

E-mail: martin.vestenicky@fel.uniza.sk

${ }^{2}$ Department of Control and Information Systems, Faculty of Electrical Engineering, University of Zilina, Slovakia,
} 


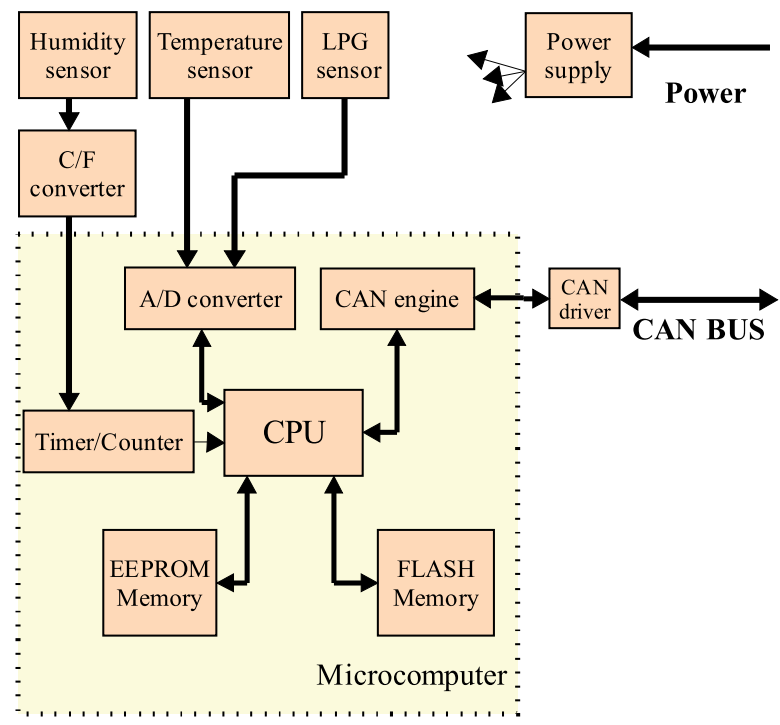

Fig. 1 Block diagram of $L P G$ sensor unit

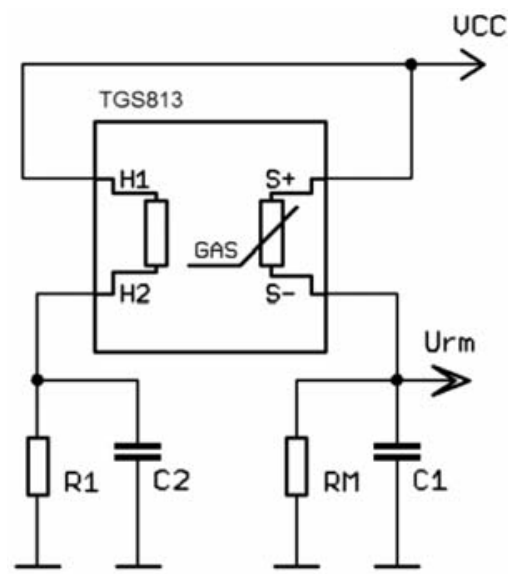

Fig. 2 Schematic diagram of the $L P G$ sensor

\section{Measurement of Relative Humidity}

For measurement of relative humidity a capacitive sensor element Philips 232269190001 was selected. Capacity $C_{S}$ of this sensor depends on the relative humidity. Inverse function of this dependence is shown in Fig. 3 (points). This dependence can be approximated by polynomial function of the second order which is given by formula (6) - curve in Fig. 3.

Measurement of the relative humidity is converted to the measurement of capacity. One of the simplest but sufficiently accurate methods of capacity measurement is utilization of measured capacity as element which determines the frequency of oscillator. In the described sensor unit the oscillator with well known CMOS timer 555 was used. The signal of astable flip - flop circuit config- ured according to Fig. 4 has duty cycle 1:1 and frequency is given by formula (7) where $a_{F}=1.4$ is constant and $R_{t}$ is timing resistance (Fig. 4). The frequency of oscillator is measured by two timers / counters which are built into used microcomputer. The first timer/counter works as counter and the second one works as time base generator. Resultant formula (8) which goes out from (6) and (7) determines the relative humidity. Values of constants for the described sensor unit are summarized in Table 1.

$$
\begin{aligned}
& R H=a_{C_{2}} \cdot C_{S}^{2}+a_{C_{1}} \cdot C_{S}+a_{C_{0}} \\
& f=\frac{1}{a_{F} \cdot R_{t} \cdot C_{S}} \\
& R H=a_{C_{2}} \cdot\left(\frac{1}{a_{F} \cdot R_{t} \cdot f}\right)^{2}+a_{C_{1}} \cdot\left(\frac{1}{a_{F} \cdot R_{t} \cdot f}\right)+a_{C_{0}}
\end{aligned}
$$

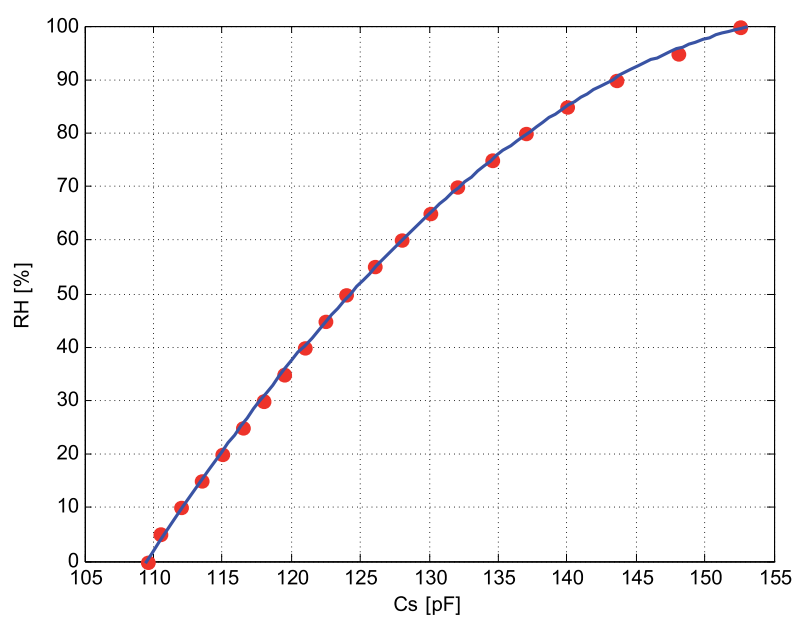

Fig. 3 Dependence of relative humidity on the sensor capacity and its approximation

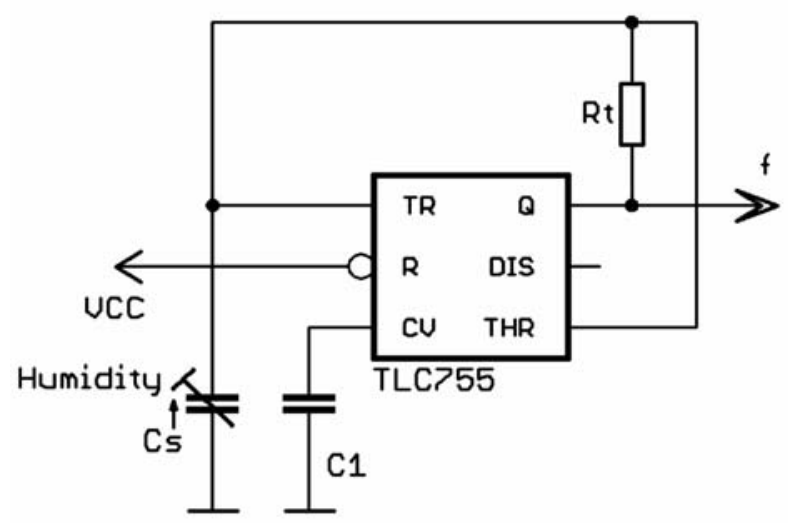

Fig. 4 Convertor of capacity to frequency 


\section{Measurement of Temperature}

The temperature is being measured by linear temperature sensor LM335 (product of National Semiconductor Corp.) whose output voltage depends on absolute temperature linearly. This dependence is described by formula (9) where $a_{T S 1}$ and $a_{T S 0}$ are constants for the given type of sensor. The temperature sensor can be calibrated by proper selection of these constants [5]. Measured temperature can be calculated from formula (10). Taking A/D process into account (11) the resultant formula for temperature calculation is (12).

$$
\begin{aligned}
& U_{T S}=a_{T S 1} \cdot T_{M E A S}+a_{T S 0} \\
& T_{M E A S}=\frac{U_{T S}-a_{T S 0}}{a_{T S 1}} \\
& U_{T S}=R E S_{A D 1} \cdot \frac{U_{C C}}{2^{r b}-1} \\
& T_{M E A S}=\frac{R E S_{A D 1} \cdot U_{C C}-\left(2^{r b}-1\right) \cdot a_{T S 0}}{\left(2^{r b}-1\right) \cdot a_{T S 1}}
\end{aligned}
$$

\section{Compensation of Temperature and Humidity Influence on the LPG Sensor}

Since the temperature and relative humidity influence the LPG sensor resistance it must be compensated. The real temperature are taken from [6]. This dependency is approximated by function of two variables $R_{H}$ and $T_{M E A S}$ according to formula (13) whereby constants for given sensor unit $a_{2}, a_{1}, a_{0}, c_{2}, c_{1}$ and $c_{0}$ are listed in Table 1. The result of approximation is depicted as curves in Fig. 5. The compensation function (14) is derived from the formula (13). Then the compensated value $R_{S 20}$ from (14) enables to calculate the LPG concentration from formula (5) if the temperature of air $T_{M E A S}$ and the relative humidity $R_{H}$ are known.

$$
\begin{aligned}
& \frac{R_{S}}{R_{S 20}}=\left(a_{2} \cdot \ln (R H+1)+c_{2}\right) \cdot T_{M E A S}^{2}+ \\
& +\left(a_{1} \cdot \ln (R H+1)+c_{1}\right) \cdot T_{M E A S}+a_{0} \cdot \ln (R H+1)+c_{0}
\end{aligned}
$$

Values of constants for LPG sensor unit

Tab. 1.

\begin{tabular}{|c|c|c|c|}
\hline$\alpha[-]$ & $K[-]$ & $a_{T S 1}\left[\mathrm{~V}^{\circ} \mathrm{C}^{-1}\right]$ & $a_{T S 0}[\mathrm{~V}]$ \\
\hline 0.5413 & 57.82 & 0.01 & 2.73 \\
\hline$a_{F}[-]$ & $r b[-]$ & $a_{C 2}\left[\mathrm{~F}^{-2}\right]$ & $a_{C 1}\left[\mathrm{~F}^{-1}\right]$ \\
\hline 1.4 & 10 & $-3.77 \cdot 10^{22}$ & $1.21908 \cdot 10^{13}$ \\
\hline$a_{C 0}[-]$ & $a_{2}\left[{ }^{\circ} \mathrm{C}^{-2}\right]$ & $c_{2}\left[{ }^{\circ} \mathrm{C}^{-2}\right]$ & $a_{1}\left[{ }^{\circ} \mathrm{C}^{-1}\right]$ \\
\hline-882.9328 & $4.382 \cdot 10-5$ & $5.359 \cdot 10-5$ & -0.003517 \\
\hline$c_{1}\left[{ }^{\circ} \mathrm{C}^{-1}\right]$ & $a_{0}[-]$ & $c_{0}[-]$ & $R_{0}[\Omega]$ \\
\hline-0.007298 & -0.1052 & 1.7970 & 1149 \\
\hline$R_{M}[\Omega]$ & $R_{t}[\Omega]$ & $U_{C C}[\mathrm{~V}]$ & \\
\hline 4700 & 8200 & 5 & \\
\hline & & & \\
\end{tabular}

$$
R_{S 20}=\frac{R_{S}}{\left(a_{2} \cdot \ln (R H+1)+c_{2}\right) \cdot T_{M E A S}^{2}+\left(a_{1} \cdot \ln (R H+1)+c_{1}\right) \cdot T_{M E A S}+a_{0} \cdot \ln (R H+1)+c_{0}}
$$

dependency of relative resistance $\left(R_{S} / R_{S 20}\right)$ of the LPG sensor with relative humidity as parameter is shown in Fig. 5 as points which

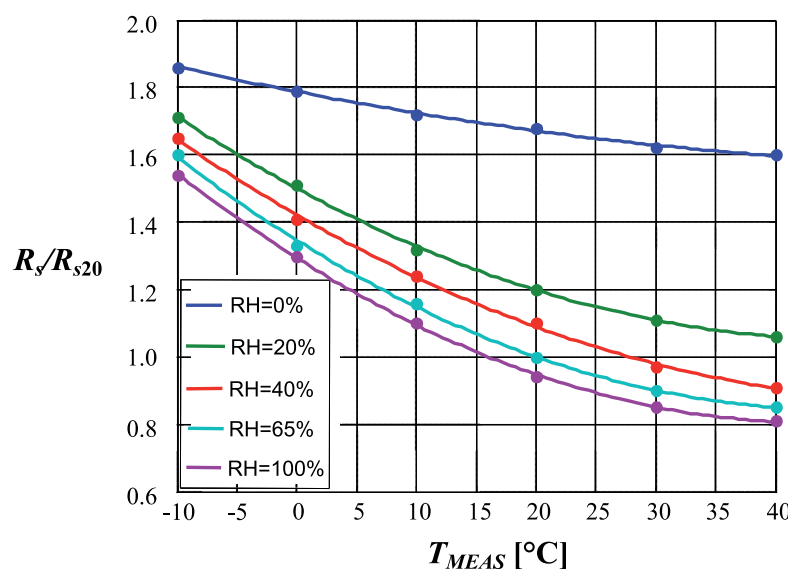

Fig. 5 Dependency of LPG sensor relative resistance on temperature and relative humidity

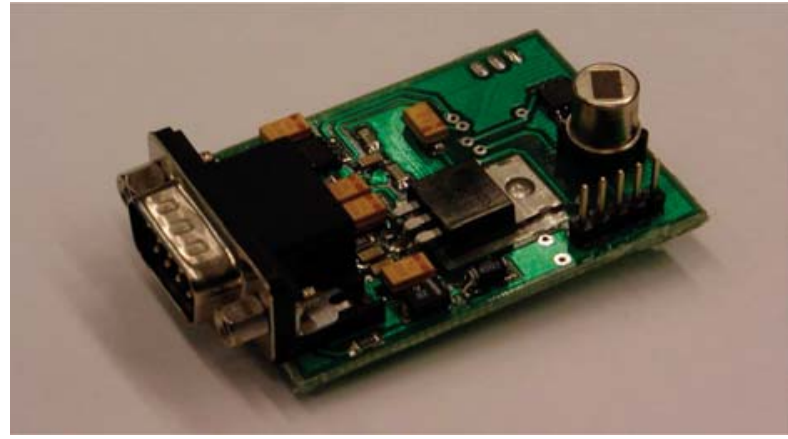

Fig. 6 Manufactured LPG sensor unit

\section{Communication Protocol on the Application Layer}

A simple communication protocol was designed for communication between sensor unit and the OBU. Standardized application protocols working on the CAN bus (CANOpen, DeviceNet) were not used for their complexity. The protocol is based on command 
Structure of the PDU

Tab. 2.

\begin{tabular}{|c|c|c|c|c|c|c|c|c|}
\hline ID & D0 & D1 & D2 & D3 & D4 & D5 & D6 & D7 \\
\hline SENS_ID & $\begin{array}{c}\text { CMD_ID/ } \\
\text { ANS_ID }\end{array}$ & MES_NBR & SENS_NBR_L & SENS_NBR_H & VAL_1_L & VAL_1_H & VAL_0_L & VAL_0_H \\
\hline
\end{tabular}

- answer principle. Protocol data units are transferred by using standard data frames with 8 octet data field in accordance to CAN 2.0A specification [4]. The PDU structure is given in Tab. 2. The CAN message identifier (11 bits) is used to address group of sensor units which measure the same quantity $(000 \mathrm{H}-\mathrm{OBU}, 010 \mathrm{H}-$ inclination sensors, $020 \mathrm{H}$ - pressure sensors, $030 \mathrm{H}$ - gas sensors, $040 \mathrm{H}$ - temperature sensors). The sensor unit uses CAN filter to select only the messages relevant for its group. The whole sensor network is addressed by message with identifier $000 \mathrm{H}$. Individual sensors can be addressed on the application layer by the 16 bits sensor unit number SENS_NBR.

The command, answer or alarm is identified by the CMD_ID/ ANS ID field (Table 3). Command parameters or measured values are transferred in the fields VAL1_L - VAL0_H. If longer message must be fragmented the field MES_NBR gives number of fragments (4 bits) and fragment order (4 bits). Fig. 7 shows an example of communication.

\section{Conclusion}

The described intelligent sensor unit (Fig. 6) was manufactured as a part of system for monitoring dangerous load transport $[1,2]$ which was solved within the international project Connect, subdomain 4.9 "Dangerous goods monitoring and information system" [3]. The method of LPG concentration measurement including compensation of temperature and relative humidity influences was implemented into firmware of the sensor unit.

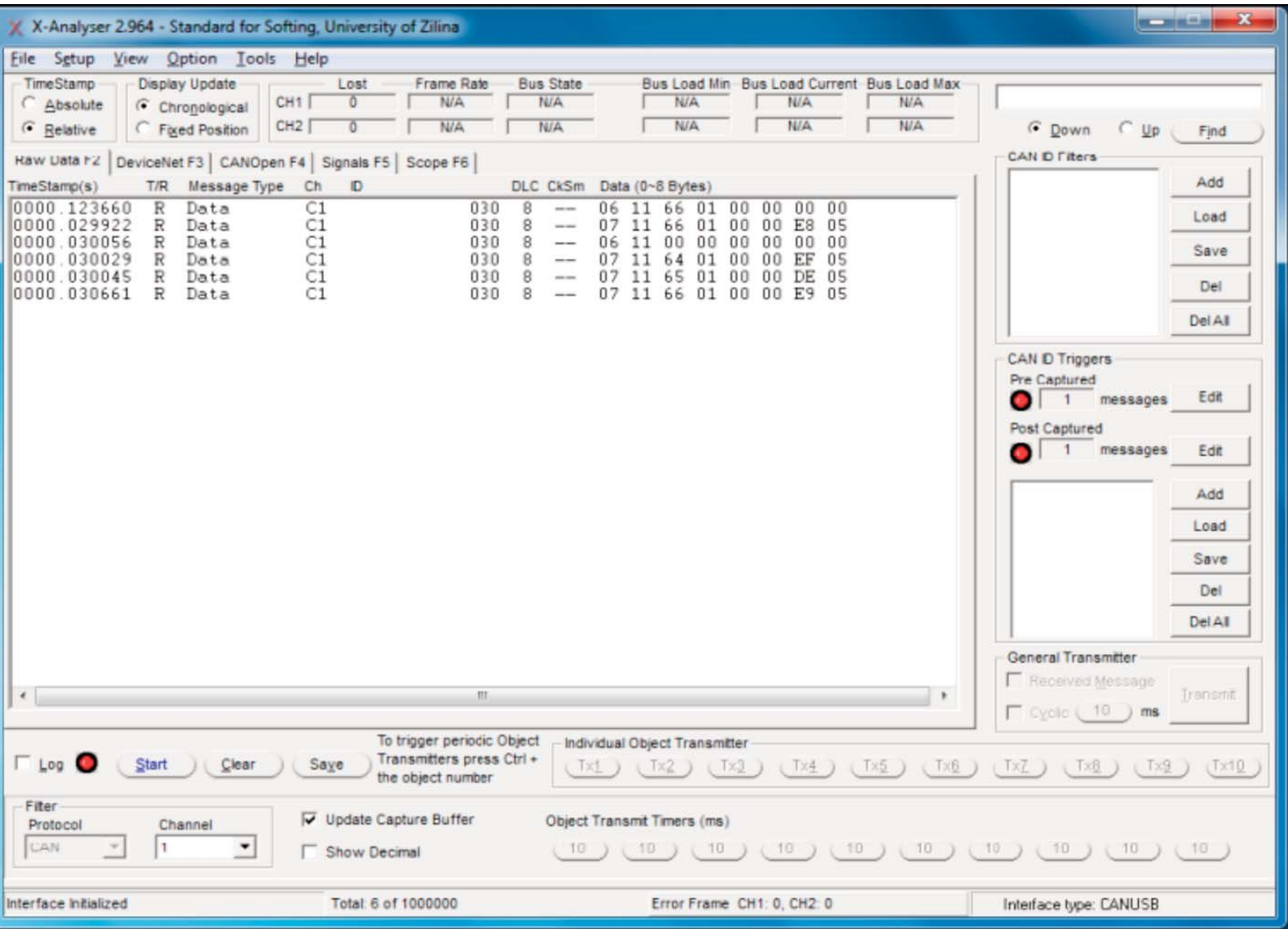

Fig. 7 Snapshot of CAN protocol analyzer 
Definition of commands, answers and alarms

\begin{tabular}{|c|c|c|}
\hline CMD_ID/ ANS_ID & TYPE & Description \\
\hline $00 \mathrm{H}$ & CMD & Get sensor unique identifier \\
\hline $01 \mathrm{H}$ & ANS & Number of sensor unit \\
\hline $02 \mathrm{H}$ & CMD & Set number of sensor unit \\
\hline $04 \mathrm{H}$ & CMD & Get sensor unit status \\
\hline $05 \mathrm{H}$ & ANS & Sensor unit status \\
\hline $06 \mathrm{H}$ & CMD & Get actual sensor data \\
\hline $07 \mathrm{H}$ & ANS & Actual sensor data \\
\hline $08 \mathrm{H}$ & CMD & Set sensor limit value \\
\hline $0 \mathrm{AH}$ & CMD & Get sensor limit value \\
\hline $\mathrm{OBH}$ & ANS & Sensor limit value \\
\hline ODH & ALM & Upper limit exceeded \\
\hline $0 \mathrm{FH}$ & ALM & Lower limit exceeded \\
\hline $11 \mathrm{H}$ & ALM & Sensor error \\
\hline $12 \mathrm{H}$ & CMD & Sensor reset \\
\hline $13 \mathrm{H}$ & ANS & OK \\
\hline $15 \mathrm{H}$ & ANS & Error \\
\hline
\end{tabular}

For the test purposes three sets of master units and sensor networks (including sensor units for measurement of pressure, inclination, and temperature) were manufactured. The tests were performed on the territory of north Slovakia by staff of Transport Research Institute, Inc. and University of Zilina. Results of the tests prove full functionality of manufactured sensor unit prototypes. Next development will be focused on design of new sensor units, for example "black box" for road traffic accident analysis.

\section{Acknowledgment}

This work was created as a part of solution of international project "Coordination and Stimulation of Innovative ITS Activities in Central and Eastern European Countries - CONNECT", TEN-T Programme EC. Project number: Addition no. 3 to Contract no. 472/ VÚD 2002.

This work has been supported by the Grant Agency VEGA of the Slovak Republic, grant No. 1/0023/08 "Theoretical Apparatus for Risk Analysis and Risk Evaluation of Transport Telematic Systems."

\section{References}

[1] VACULIK, M., FRANEKOVA, M., VESTENICKY, P., VESTENICKY, M.: On-Board Unit and its Possibilities of Communications on Safety and Security Principles. AEEE 7, 2008, No. 1-2, pp. 235-238. ISSN 1336 - 1376

[2] VACULIK, M., VESTENICKY, P., VESTENICKY, M.: Vehicle On-board Unit (in Slovak). Proc. of Int'l conference ITS, Bratislava, 2007, ISBN 978-80-254-0207-8

[3] CONNECT, Co-ordination and Stimulation of Innovative ITS Activities in Central and Eastern European Countries. [online]. URL $<$ http://www.connect-project.org/>

[4] CAN specification, Version 2.0. Robert Bosch Gmbh. 1991. [online] URL 〈http://www.semiconductors.bosch.de/pdf/can2spec.pdf〉

[5] BLAGOJEVIC, M., PETKOVIC, D.: A New Approach to the Interpretation of Signals from Temperature Sensors. Communications - Scientific Letters of University of Zilina, Vol. 8, Nr. 4, 2006, pp. 57-60. ISSN 1335-4205.

[6] Figaro product information: TGS 813 - for the detection of Combustible Gases, rev. 9/02. [online] URL http://www.figarosensor.com/products/813pdf.pdf. 